\title{
QUANTUM MECHANICS, RANDOMNESS, AND DETERMINISTIC REALITY
}

\author{
Detlef Dürr ${ }^{(a)}$, Sheldon Goldstein And Nino Zanghí ${ }^{(b)}$ \\ Department of Mathematics \\ Rutgers University \\ New Brunswick, NJ 08903
}

\begin{abstract}
We describe and analyze a new formulation of Bohmian mechanics - the deterministic theory of particles in motion that emerges from Schrödinger's equation for a system of particles when we merely insist that "particles" means particles. This mechanics resolves all paradoxes associated with the measurement problem in nonrelativistic quantum mechanics. It accounts for quantum randomness, absolute uncertainty, the meaning of the wave function of a system, collapse of the wave function, and familiar (macroscopic) reality.
\end{abstract}

PACS numbers: 03.65.Bz

\section{INTRODUCTION}

According to the quantum formalism, measurements performed on a quantum system with definite wave function typically yield random results. However, the quantum dynamics governing the evolution of the wave function over time, at least when no measurement is being performed, and given, say, by Schrödinger's equation, is completely deterministic. Thus if all physical processes, including those we call measurements, are governed by the same fundamental laws, and if one assumes the wave function to be

July 24, 2004

${ }^{(a)}$ Current address: Fakultät für Mathematik, Universität München, Theresienstrasse 39, 8000 München 2, Germany.

${ }^{(b)}$ Current address: Istituto di Fisica, Università di Genova, INFN, Via Dodecaneso 33, 16146 Genova, Italy. 
the complete description of the physical state, one arrives at an inconsistency, often referred to as "the measurement problem" or the problem of the "collapse of the wave function."

Debates over this problem have been strongly influenced by "quantum epistemology" - by the obligatory appeal to the observer in a measurement, whose act of "observation" somehow creates the random outcome. In recent years, however, the growing disenchantment with this irreducible reference to the observer has begun to assume the form of serious doubts (see, e.g., [?]) about the strict validity and meaning of the quantum formalism - with several recent proposals [2-7] explicitly suggesting the abrogation of the (unitary) Schrödinger evolution, and with proposals for various experimental tests, some of which have been carried out.

Of a quite different character are the proposals $[?, ?, ?]$ which, while leaving this evolution law intact, seek an ontological basis for the formalism; i.e., they encourage the search for basic entities which have so far been left out of the description, but which are in fact the source of the appearance of "real events" like the outcomes of measurements. Here the wave function attains physical significance via its relation to the behavior of something else more concretely objective.

Now when the question of ontology is raised, the no-hidden-variables theorems [?] spring to mind. These are frequently misinterpreted as precluding the possibility of explaining quantum randomness by averaging over ignorance. They have been used to support the radical claim that we must abandon any pretension of describing nature in objective terms - of attempting to construct a fully objective microscopic theory from which the quantum formalism will emerge, as a phenomenological formalism describing certain macroscopic regularities.

It is thus somewhat surprising that such a theory was constructed by David Bohm [?] in 1952, who developed it from the guiding field idea of de Broglie [?]. For Bohm it is particles, described by their positions in space, which form the basis of the ontology. The wave function choreographs their motion. Thus for Bohm the complete state of an $N$-particle quantum system is given by the configuration $\mathbf{Q}_{1}, \ldots, \mathbf{Q}_{N}$ of its particles 
and by its wave function $\psi$.

Unfortunately, this theory was presented in close analogy with Newtonian mechanics (in its Hamilton-Jacobi formulation), in such a way that it seemed artificial - rather than as the radically non-Newtonian mechanics that it is. Our formulation (see below) of Bohm's theory - which we call Bohmian mechanics - makes transparent that Bohmian mechanics is a new mechanics, a completely deterministic, but distinctly nonNewtonian, theory of particles in motion. Moreover, while its formulation does not involve the notion of quantum observables as self-adjoint operators - so that its relationship to the quantum formalism may at first appear somewhat obscure - it can in fact be shown that the theory not only accounts for quantum phenomena $[?, ?, ?, ?]$, but also embodies the quantum formalism itself as the very expression of its empirical import [?,?].

That this is so is for the most part quite straightforward. It is based in part on what is now often referred to as decoherence - the ever decreasing possibility of interference between, e.g., macroscopically distinct wave functions due to (typically uncontrollable) interactions with, e.g., the environment [?,?,?,?]. More fundamentally, however, it involves a crucial subtlety concerning the randomness of quantum phenomena. It is easy to see that the predictions of Bohmian mechanics for the results of a quantum experiment on a system having wave function $\psi$ are precisely those of the quantum formalism, provided it is assumed that prior to the experiment the particle configuration of the system is randomly distributed according to Born's statistical law, i.e., according to the probability density $\rho=|\psi|^{2}$. But how, in this deterministic theory, does randomness enter? What is special about $\rho=|\psi|^{2}$ ? And precisely to which ensemble does this probability distribution refer?

To come to grips with these questions, we need first to formulate sharply and then to analyze carefully Bohmian mechanics. The most striking feature which emerges is the nonseparability of the theory; as a consequence, the applicability of Bohmian mechanics to a subsystem of a larger system governed by Bohmian mechanics is not a matter of choice but a question of fact. When this is taken into account our difficulty is 
dissolved, by careful scrutiny of the relevant concepts - most notably those of quantum equilibrium and of the effective wave function of a subsystem.

We wish to call particular attention to an immediate consequence of our definition of effective wave function: the fundamental conditional probability formula (7), a formula whose simplicity and superficial familiarity tend to obscure both its power and its true novelty. From this formula it follows, almost at once, that a Bohmian universe, though deterministic, evolves in such a manner that an appearance of randomness emerges, with empirical distributions in agreement with the predictions of the quantum formalism.

In concluding this introduction, we feel obliged to emphasize, both for those who yearn for a return by physics to its classical past as well as for some proponents of Copenhagen orthodoxy, that classical physics is not a priori ${ }^{1}$ - that its abandonment does not demand a repudiation of realism - and that a scientific explanation, of the behavior of objective entities, does not mean only an account based on the concepts of classical physics. It should not be necessary, some sixty years after the quantum revolution, to say these things, but it is!

Note, however, that the most prominent antagonist of quantum orthodoxy, a physicist commonly perceived as stubbornly refusing to abandon the classical world-view, has said that the difficulties of quantum mechanics

are connected with the fact that one retains the classical concepts of force or potential energy.... To me it seems, however, that one will finally recognize that something must take the place of forces acting....(Einstein, in [?])

\section{BOHMIAN MECHANICS}

Consider a quantum system of $N$ particles with masses $m_{1}, \ldots, m_{N}$, generic configuration space variables $q=\left(\mathbf{q}_{1}, \ldots, \mathbf{q}_{N}\right)$, and wave function $\psi=\psi(q)$. Bohmian mechanics is the (simplest) theory of motion for these particles determined by a firstorder time-reversal and Galilean invariant guiding law (see [?]). The state of the system

\footnotetext{
${ }^{1}$ See, for example, [?] to the contrary.
} 
is given by $(Q, \psi)$, where $Q=\left(\mathbf{Q}_{1}, \ldots, \mathbf{Q}_{N}\right)$ is the actual configuration of the particles and $\psi$ satisfies Schrödinger's equation. The configuration $Q$ evolves through a first-order evolution equation, and the role of $\psi$ is to generate a (velocity) vector field $v^{\psi}=\left(\mathbf{v}_{1}^{\psi}, \ldots, \mathbf{v}_{N}^{\psi}\right)$ on configuration space which governs this evolution. Covariance determines the form of $v^{\psi}$, and one thus arrives naturally at the evolution law for

\section{Bohmian mechanics,}

$$
\begin{gathered}
\frac{d \mathbf{Q}_{k}}{d t}=\mathbf{v}_{k}^{\psi}\left(\mathbf{Q}_{1}, \ldots, \mathbf{Q}_{N}\right) \equiv \frac{\hbar}{m_{k}} \operatorname{Im} \frac{\boldsymbol{\nabla}_{\mathbf{q}_{k}} \psi}{\psi}\left(\mathbf{Q}_{1}, \ldots, \mathbf{Q}_{N}\right), \\
i \hbar \frac{\partial \psi}{\partial t}=-\sum_{k=1}^{N} \frac{\hbar^{2}}{2 m_{k}} \nabla_{\mathbf{q}_{k}}^{2} \psi+V \psi .
\end{gathered}
$$

Before beginning our analyis of the origin of randomness in a Bohmian universe, we wish to pause briefly to emphasize two points. First, Bohmian mechanics is completely defined by (1) and (2) (together with the specification of $V$ ). In particular, there is no need - and, indeed, no room — for any "measurement postulates," or for axioms governing the behavior of "other observables": Any such specification would at best be redundant, and would quite possibly be inconsistent.

Secondly, the reader familiar with Bohm's theory should note that the quantum potential, introduced and emphasized by Bohm (see [?] and [?]) — but repeatedly dismissed, by omission, by Bell (see [?]) — does not appear in the above formulation of Bohmian mechanics. Moreover, since it is a "first-order theory," the second-order (Newtonian) concepts of acceleration and force, work and energy do not play a fundamental role in Bohmian mechanics. ${ }^{2}$ [From the perspective of Bohmian mechanics, Schrödinger's equation is, in fact, best regarded as merely the simplest choice of covariant equation for the guiding field $\psi$ (see [?] and [?]), with no particular a priori interpretation of the parameters, such as the "masses" or the function $V$, which appear

\footnotetext{
${ }^{2}$ This is not to say that these concepts play no role in Bohmian mechanics; they are emergent notions, fundamental to the theory to which Bohmian mechanics converges in the "classical limit," namely, Newtonian mechanics. Moreover, in order to analyze this limit, it is extremely convenient to transform the defining equations (1) and (2) of Bohmian mechanics into Bohm's original HamiltonJacobi form involving the quantum potential. One then sees that the (size of the) quantum potential provides a measure of the deviation of Bohmian mechanics from its classical approximation.
} 
in it.] From our perpective the artificiality suggested by the quantum potential is the price one pays if one insists on casting a highly nonclassical theory into a classical mold.

\section{THE ORIGIN OF QUANTUM RANDOMNESS}

\subsection{Quantum equilibrium.}

We now begin our analysis of Bohmian mechanics. We first remark that it is important to recognize that an $(n$-particle, $n<N)$ subsystem need not in general be governed by Bohmian mechanics, since no wave function for the subsystem need exist. This will be so, even with trivial interaction potential $V$, if the wave function does not properly factorize; for nontrivial $V$ the Schrödinger evolution would in any case quickly destroy such a factorization.

Therefore in a universe governed by Bohmian mechanics there is a priori only one wave function, namely that of the universe, as there is a priori only one system governed by Bohmian mechanics, namely the universe itself.

We shall return shortly to these considerations, but wish now to focus on the clear need - since our primary concern is with the origin of randomness - for a qualitative statistical analysis, roughly analogous to that of statistical mechanics, where the stationary (Liouville) measure on phase space plays an important role. Note that since $\psi$ will in general be time-dependent, the vector field $v^{\psi}$ will be time-dependent as well, and we cannot expect the evolution on configuration space to possess a stationary distribution.

However, we may ask for a distribution stationary relative to $\psi$, playing essentially the same role as a stationary one: An ensemble density $\rho(q, t)$ on configuration space evolving under the solution flow of (1), i.e., satisfying the continuity equation

$$
\frac{\partial \rho}{\partial t}+\operatorname{div}\left(\rho v^{\psi}\right)=0
$$

and given by a functional of $\psi$, is said to be equivariant if it retains its form as a functional of $\psi$. An equivariant density is provided by $|\psi|^{2}$, as one easily checks by noting that $J^{\psi}=|\psi|^{2} v^{\psi}$, where $J^{\psi}=\left(\mathbf{J}_{1}^{\psi}, \ldots, \mathbf{J}_{N}^{\psi}\right)$ is the quantum probability current. In other words, if $\rho\left(q, t_{0}\right)=\left|\psi\left(q, t_{0}\right)\right|^{2}$ at some time $t_{0}$, then $\rho(q, t)=|\psi(q, t)|^{2}$ for all $t$. 
We call the probability distribution on configuration space given by $\rho \equiv|\psi|^{2}$ the quantum equilibrium distribution, and say that a system is in quantum equilibrium if its configuration is so distributed. We may now reformulate our earlier questions concerning quantum randomness as seeking the clarification and justification of the quantum equilibrium hypothesis: When a system has wave function $\psi$, the distribution $\rho$ of its configuration satisfies $\rho=|\psi|^{2}$. Using equivariance, the quantum formalism emerges rather straightforwardly from the quantum equilibrium hypothesis, the latter thus embodying the empirical import of the theory.

However, in accordance with an earlier observation, in a Bohmian universe there can be a priori only one system in quantum equilibrium, namely, the universe itself. And this statement, which to some may sound rather innocent, in fact embodies part of the subtlety to which we have earlier referred. Of universes, we have only one-ours - at our disposal. What possible physical significance can be attached to a quantum equilibrium ensemble of universes?

There is a rather simple answer. The quantum equilibrium distribution provides us with a natural notion of typicality: statements valid for the overwhelming majority of configurations in the sense provided by the quantum equilibrium measure are true for a typical configuration. (Just as in statistical mechanics and dynamical systems theory, the notion of typicality is somewhat delicate. In particular, the previous sentence should be regarded as conveying only what is meant by saying that something is true for typical configurations, without, however, precisely specifying which configurations are in fact typical. Such a specification would be meaningful only relative to a given set of properties.) Note that by equivariance this notion of typicality is time-independent. However, it is best to regard it as applied to initial universal configurations, at the time of "creation" or shortly thereafter.

We establish the remarkable fact that the quantum equilibrium hypothesis, properly understood, is a simple manifestation of universal quantum equilibrium, in the sense of typicality. And if we are to make contact with physics, i.e., to arrive at this proper understanding of $\rho=|\psi|^{2}$, we must relate $\rho$ to the empirical distribution of configurations 
arising from repetitions of similar experiments, performed at different places or times within a single typical sample of the universe (such as ours). But before doing so, we must first come to grips with a problem hinted at earlier, that of the notion of the wave function of a subsystem.

\subsection{The effective wave function.}

Consider an $N$-particle nonrelativistic universe governed by Bohmian mechanics, with (universal) wave function now denoted by $\Psi$. Focus on a subsystem with configuration variables $x$, i.e., on a splitting $q=(x, y)$ where $y$ represents the configuration of the environment of the $x$-system. The actual particle configurations are accordingly denoted by $X$ and $Y$, i.e., $Q=(X, Y)$. Note that $\Psi=\Psi(x, y)$.

How can one assign a wave function to the $x$-system? In orthodox quantum theory the question touches metaphysical ground, insofar as its answer would refer to an observer. How does Bohmian mechanics handle this question?

One obvious possibility-afforded by the existence of the actual configuration-is given by what we call the conditional wave function

$$
\psi(x)=\Psi(x, Y)
$$

(Nonvanishing scalar multiples of wave functions are identified.) Note that by virtue of (1) the velocity vector field for the $x$-system is determined by its conditional wave function. This, however, does not in general have the usual dynamical significance of the wave function of a system, as it does not in general evolve according to Schrödinger's equation, even when the $x$-system is dynamically decoupled from its environment.

But one can go further, exploring the form of the universal wave function-a superposition of macroscopically distinct terms - which according to standard quantum measurement theory should arise from a measurement on the $x$-system. One then recognizes that the following definition (see [?]) captures all the desirable phenomenological aspects (through its reference to the "macroscopic"): Suppose that

$$
\Psi(x, y)=\psi(x) \Phi(y)+\Psi^{\perp}(x, y),
$$


where $\Phi$ and $\Psi^{\perp}$ have macroscopically disjoint $y$-supports. If

$$
Y \in \operatorname{supp} \Phi
$$

we say that $\psi$ is the effective wave function of the $x$-system.

The effective wave function is indeed a "collapsed" wave function, and agrees with the wave function assigned to the $x$-system by the quantum formalism (to the extent that the latter does assign a wave function), as well as with the conditional wave function. Note also that the fact that the (conditional or effective) wave function of a subsystem depends critically on the actual environment $Y$, in which all knowledge is grounded, is not unrelated to the epistemological component - the appeal to the observer - with which the wave function has traditionally been entangled.

\subsection{Quantum randomness and absolute uncertainty.}

We wish now to explain how Born's statistical law arises. For this consider-for the moment solely as a mathematical device - the probability space of initial universal configurations $Q$ distributed according to the quantum equilibrium distribution $\mathbf{P}^{\Psi_{0}}(d Q)=\left|\Psi_{0}(Q)\right|^{2} d Q$ - the only natural measure available - where $\Psi_{0}$ is the initial universal wave function. Let $Q_{t}$ and $\Psi_{t}$ denote the universal configuration and the universal wave function at time $t$. Since $\left(Q_{t}, \Psi_{t}\right)$ satisfies the Bohmian evolution (1)(2), subject to the initial conditions given by $Q$ and $\Psi_{0}$, it follows that, for given $\Psi_{0}$, $Q_{t}=\left(X_{t}, Y_{t}\right)$ is a function of the initial universal configuration $Q$ and as such is a random variable.

Note that by equivariance the distribution of $Q_{t}$ is given by $\left|\Psi_{t}\right|^{2}$. It thus follows at once from the definition of the (effective or conditional) wave function that the conditional distribution of the configuration of the $x$-system, given the configuration of its environment, is given by

$$
\mathbf{P}^{\Psi_{0}}\left(X_{t} \in d x \mid Y_{t}\right)=\left|\psi_{t}(x)\right|^{2} d x
$$

where $\psi_{t}$ is the wave function of the $x$-system at time $t$. 
Suppose now that at time $t$ the $x$-system consists itself of many identical subsystems $x_{1}, \ldots, x_{M}$, each one having effective wave function $\psi$ (with respect to coordinates relative to suitable frames). Then the effective wave function of the $x$-system is the product wave function $\psi_{t}(x)=\psi\left(x_{1}\right) \cdots \psi\left(x_{M}\right)$ (see [?]). It follows immediately from (7) that, given the configuration $Y_{t}$ of the environment of the $x$-system, the configurations $X_{1}, \ldots, X_{M}$ of the subsystems at time $t$ form a collection of independent random variables, identically distributed, with common distribution $\rho_{q e}=|\psi|^{2}$.

We emphasize that the previous statement is, per se, devoid of physical significance, implicitly referring, as it does, to the probability space given by $\mathbf{P}^{\Psi_{0}}$, i.e., to an ensemble of universes. But we may now easily make contact with physics: The weak law of large numbers yields that for large $M$ the empirical distribution of the configurations will be well approximated by $\rho_{q e}$ for the $\left(\mathbf{P}_{Y_{t}}^{\Psi_{0}}\right)$ overwhelming majority of initial universal configurations $Q$ (where $\mathbf{P}_{Y_{t}}^{\Psi_{0}}(d Q)=\mathbf{P}^{\Psi_{0}}\left(d Q \mid Y_{t}\right)$ ). In other words, for typical initial configurations of the universe (consistent with the environment of the $x$-system at time t) the empirical distribution is given by Born's law.

Of course, Born's statistical law is supposed to govern not only equal-time ensembles, but also ensembles referring to experiments performed on one or more (sub)systems at possibly different times. In order to deal with this general situation, we are forced (see [?]) to consider a random system $\sigma=(\pi, T)$, i.e., a random subsystem at a random time and satisfying a certain measurability condition. We find that a random system version of (7) —analogous to a strong Markov property-remains valid, permitting a general random system analysis leading to empirical distributions in complete agreement with the predictions of the quantum formalism. It turns out that in this case the "recording" of the "results of measurement" plays a crucial role.

The most remarkable and perhaps most surprising consequence of our analysis is what we have called absolute uncertainty: Notice that (7) involves conditioning on the detailed microscopic state of the environment, in which the results of measurements and, indeed, all our information - and far more than we could ever reasonably have access to - is grounded. It follows from (7) that in a universe governed by Bohmian mechanics, 
it is in principle impossible to know more about the configuration of a subsystem than what is expressed by the quantum equilibrium hypothesis (see [?]).

When all is said and done, quantum randomness and quantum uncertainty are merely an expression of quantum equilibrium, a global configurational equilibrium subordinate to the universal (and, in fact, nonequilibrium) wave function $\Psi$ (see [?]).

\section{Concluding Comments}

We remark that, as with statistical mechanics and as a consequence of quantum equilibrium, integration of the Bohmian evolution equation (1) (for, say, simple models) has little or no predictive value, despite what at first might have been expected. Notice, however, that this in no way diminishes the physical significance of this evolution. (See also [?].)

For clarity we have restricted our attention to particles without spin. We remark that spin and the exclusion principle enter through the choice of appropriate wave function and corresponding guiding law $[?, ?, ?, ?, ?]$.

The extension of Bohmian mechanics to a relativistic quantum theory has proven difficult. We do feel, however, that a major virtue of the kind of physics explored here is its flexibility, which may permit the opening of new paths towards an understanding of the content of such a theory [?]. In particular, we note that our analysis does not depend crucially on a particle ontology.

For a recent article expressing concerns similar to those of the present paper, see [?]. We note, however, that the analysis of the present paper shows that that of [?] — even if it were mathematically correct-is neither necessary nor sufficient!

We gratefully acknowledge useful comments and suggestions by K. Berndl, J. T. Cushing, M. Daumer, G. Eyink, P. Garrido, R. Goldstein, A. Kupiainen, H. Sussmann, and especially by J. L. Lebowitz and E. Speer. This work was supported in part by National Science Foundation Grant No. DMS-9105661, by DFG, and by INFN.

\section{REFERENCES}

1. J. S. Bell, Speakable and unspeakable in quantum mechanics, Cambridge University Press, Cam- 
bridge, 1987.

2. E. P. Wigner, Quantum Optics, Experimental Gravity and Measurement Theory (P. Meystre and M. O. Scully, eds.), Plenum, New York, 1983, pp. 43-63.

3. A. J. Leggett, Prog. Theor. Phys. Supplt. 69 (1980), 80.

4. H. P. Stapp, Quantum Implications (B. J. Hiley and F. D. Peat, eds.), Routledge \& Kegan Paul, London, 1987, pp. 255-266.

5. S. Weinberg, Phys. Rev. Lett. 62 (1989), 485.

6. R. Penrose, Quantum Concepts in Space and Time (R. Penrose and C. J. Isham, eds.), Oxford University Press, Oxford, 1985, see also [?].

7. G. C. Ghirardi, A. Rimini, and T. Weber, Phys. Rev. D 34 (1986), 470.

8. D. Bohm, Phys. Rev. 85 (1952), 166-193.

9. E. Nelson, Quantum Fluctuations, Princeton University Press, Princeton, N.J., 1985.

10. M. Gell-Mann and J. B. Hartle, Complexity, Entropy, and the Physics of Information (W. Zurek, ed.), Addison-Wesley, Reading, 1990, pp. 425-458.

11. J. S. Bell, Rev. Mod. Phys. 38 (1966), 447.

12. L. de Broglie, La Nouvelle Dynamique des Quanta, Electrons et Photons, Gauthier-Villars, Paris, 1928, pp. 105-132.

13. D. Bohm and B. Hiley, The Undivided Universe: an Ontological Interpretation of Quantum Theory, Routledge \& Kegan Paul, London, 1992.

14. M. Daumer, D. Dürr, S. Goldstein, and N. Zanghí, On the Role of Operators in Quantum theory, in preparation.

15. M. Daumer, D. Dürr, S. Goldstein, and N. Zanghí, The Mystery of Quantization, in preparation.

16. W. H. Zurek, Phys. Rev. D 26 (1982), 1862.

17. E. Joos and H. D. Zeh, Zeit. Phys. B 59 (1985), 223.

18. W. Heisenberg, Physics and Philosophy, Harper and Row, New York, 1958, p. 97.

19. A. Einstein, Louis de Broglie, Physicien et Penseur (A. George, ed.), Éditions Albin Michel, Paris, 1953, quoted in [?].

20. J. Stachel, Einstein and the Quantum, Fifty Years of Struggle, From Quarks to Quasars (R. G. Colodny, ed.), University of Pittsburgh Press, Pittsburgh, 1986. 
21. D. Dürr, S. Goldstein, and N. Zanghí, Quantum Equilibrium and the Origin of Absolute Uncertainty, J. Stat. Phys. 67 (1992), 843-907.

22. J. Levy-Leblond, J. Math. Phys. 4 (1963), 776-788.

23. D. Dürr, S. Goldstein, and N. Zanghí, Quantum Chaos, Classical Randomness, and Bohmian Mechanics, J. Stat. Phys. 68 (1992), 259-270.

24. D. Dürr, S. Goldstein, and N. Zanghí, First-order Theories, in preparation.

25. S. Goldstein, J. Stat. Phys. 47 (1987), 645.

26. D. Dürr, S. Goldstein, and N. Zanghí, Stochastic Processes, Geometry and Physics (S. Albeverio, G. Casati, U. Cattaneo, D. Merlini, R. Mortesi, eds.), World Scientific, Singapore, 1990, pp. 374391.

27. A. Valentini, Phys. Lett. A 156 (1991), 5.

28. R. Penrose, The Emperor's New Mind, Oxford University Press, New York and Oxford, 1989. 